\title{
ERRATUM
}

Thomas J. Feuerstein · Norbert Limberger

\section{Mathematical analysis of the control of neurotransmitter release by presynaptic receptors as a supplement to experimental data}

Published online: 10 September 1999

Naunyn-Schmiedeberg's Arch Pharmacol (1999) 359:345-359

Due to an unfortunate oversight, the address of N. Limberger was given incorrectly. The correct address is as follows: Pharmakologisches Institut, Hermann-Herder-Straße 5, Universität Freiburg, D-79104 Freiburg, Germany.

The online version of the original article can be found at http://link.springer.de/link/service/journals/00210/bibs/9359005/ 93590345.htm

T. J. Feuerstein (ㄹ)

Sektion Klinische Neuropharmakologie, Neurozentrum,

Universität Freiburg, Breisacherstraße 64,

D-79106 Freiburg, Germany

e-mail: feuer@ukl.uni-freiburg.de,

Fax: +49-761-2705281

N. Limberger

Pharmakologisches Institut, Hermann-Herder-Straße 5,

Universität Freiburg, D-79104 Freiburg, Germany 